\title{
Pilot study of intracranial venous physiology in craniosynostosis
}

\author{
Martijn J. Cornelissen, MD, PhD, ${ }^{1}$ Robbin de Goederen, MD, ${ }^{1}$ Priya Doerga, MD, ${ }^{1}$ \\ Iris Cuperus, BSc, ${ }^{1}$ Marie-Lise van Veelen, MD, PhD, ${ }^{2}$ Maarten Lequin, MD, PhD, ${ }^{3}$ \\ Paul Govaert, MD, PhD, ${ }^{4}$ Irene M. J. Mathijssen, MD, PhD, MBA-H, ${ }^{1}$ Jeroen Dudink, MD, PhD, ${ }^{5}$ and \\ Robert C. Tasker, MA, MD, FRCP(UK) ${ }^{6}$
}

\begin{abstract}
Departments of ${ }^{1}$ Plastic and Reconstructive Surgery and Hand Surgery and ${ }^{2}$ Neurosurgery, Sophia Children's Hospital, Erasmus MC, University Medical Center, Rotterdam; ${ }^{3}$ Department of Radiology and 5 Neonatology Division, Department of Pediatrics, Wilhelmina Children's Hospital, University Medical Center, Utrecht, The Netherlands; ${ }^{4}$ Neonatology Division, Department of Pediatrics, Koningin Paola Children's Hospital, Antwerp, Belgium; and 'Departments of Neurology and Anesthesia (Pediatrics), Harvard Medical School, Boston Children's Hospital, Boston, Massachusetts
\end{abstract}

\begin{abstract}
OBJECTIVE In addition to craniocerebral disproportion, other factors, such as Chiari malformation type I, obstructive sleep apnea, and venous outflow obstruction, are considered to have a role in the occurrence of intracranial hypertension in craniosynostosis. This pilot study examined cerebral venous flow velocity to better characterize the complex intracranial venous physiology of craniosynostosis.
\end{abstract}

METHODS The authors performed a prospective cohort study of craniosynostosis patients $(n=34)$ referred to a single national (tertiary) craniofacial unit. Controls $(n=28)$ consisted of children who were referred to the unit's outpatient clinic and did not have craniosynostosis. Transfontanelle ultrasound scans with venous Doppler flow velocity assessment were performed at the first outpatient clinic visit and after each surgery, if applicable. Mean venous blood flow velocities of the internal cerebral vein $\left(\mathrm{ICV}_{\mathrm{v}}\right)$ and the superior sagittal sinus $\left(\mathrm{SSS}_{\mathrm{v}}\right)$ were recorded and blood flow waveform was scored.

RESULTS Preoperatively, SSS was decreased in craniosynostosis patients compared with controls $(7.57 \mathrm{vs} 11.31 \mathrm{~cm} /$ $\mathrm{sec}, p=0.009)$. ICV $\mathrm{v}_{\mathrm{v}}$ did not differ significantly between patients and controls. Postoperatively, $\mathrm{SSS}_{\mathrm{v}}$ increased significantly $(7.99 \mathrm{vs} 10.66 \mathrm{~cm} / \mathrm{sec}, p=0.023)$. Blood flow waveform analyses did not differ significantly between patients and controls.

CONCLUSIONS Premature closure of cranial sutures was associated with decreased $\mathrm{SSS}_{\mathrm{v}}$ but not ICV ${ }_{\mathrm{v}}$; indicating an effect on the superficial rather than deep venous drainage. Further Doppler ultrasound studies are needed to test the hypothesis that at an early stage of craniosynostosis pathology $\mathrm{SSS}_{\mathrm{v}}$, but not pulsatility, is abnormal, and that abnormality in both SSS $_{v}$ and the superficial venous waveform reflect a more advanced stage of evolution in suture closure.

https://thejns.org/doi/abs/10.3171/2018.1.PEDS17480

KEYWORDS intracranial hypertension; craniosynostosis; Doppler ultrasound; superior sagittal sinus; cerebral venous drainage; craniofacial

RANIOSYNOSTOSIS occurs in approximately 1 in 1500 births and results in abnormal shape of the cranium and increased risk of intracranial hypertension (ICHT). ${ }^{1}$ In published series, the prevalence of ICHT ranges from $1 \%$ to $85 \%$, and it is particularly high in syndromic cases of craniosynostosis. ${ }^{2,3,13,17}$ Historically, the development of ICHT in craniosynostosis was attributed solely to skull growth restriction (i.e., craniocerebral disproportion)..$^{18}$ Now, however, the accumulated evidence suggests that other factors may also be relevant, ${ }^{5,6,8,9,19}$ including: cranial vault venous outflow obstruction, ventriculomegaly (or hydrocephalus if progressive), tonsillar herniation or presence of Chiari malformation type I, and obstructive sleep apnea (OSA). These pathophysiological features are

ABBREVIATIONS CSF = cerebrospinal fluid; ICHT = intracranial hypertension; ICP = intracranial pressure; ICV = internal cerebral vein; ICV $=$ mean venous blood flow velocity of the ICV; MANOVA = multivariate analysis of variance; OFC = occipitofrontal head circumference; OSA = obstructive sleep apnea; $P_{S S S}=S S S$ pressure; SSS = superior sagittal sinus; SSS $_{\mathrm{v}}=$ mean venous blood flow velocity of the SSS.

SUBMITTED August 27, 2017. ACCEPTED January 11, 2018.

INCLUDE WHEN CITING Published online April 6, 2018; DOI: 10.3171/2018.1.PEDS17480. 
rarely seen in single-suture craniosynostosis patients, and so we have to conclude that they are unlikely to account for the development of ICHT in such patients. In our previous work we have recognized a discrepancy in the rate of ICHT by suture involvement that is not readily explained by any of the mechanisms outlined above. For example, in cases of metopic suture synostosis, the rate of ICHT is low $(1 \%-2 \%)$, irrespective of relatively small intracranial volume after surgery. ${ }^{2,12}$ The opposite is true in sagittal suture synostosis; that is, despite a relatively larger intracranial volume, ICHT is found in $6 \%-10 \%$ of patients. ${ }^{7,20}$

In this context, clinical researchers have focused on cerebral venous drainage in craniosynostosis, albeit with few definitive studies. We know that there is an interaction between superior sagittal sinus $(\mathrm{SSS})$ pressure $\left(\mathrm{P}_{\mathrm{SSS}}\right)$ and intracranial pressure (ICP). For example, as early as 1984, Sainte-Rose et al. suggested that a rise in $\mathrm{P}_{\text {SsS }}$ due to obstruction resulted in a rise in ICP. ${ }^{19}$ We also know that the mean venous blood flow velocity of the SSS $\left(\mathrm{SSS}_{\mathrm{v}}\right)$, measured using Doppler ultrasound in single-suture cases of craniosynostosis, differs from the norm. ${ }^{15}$ Last, we know that cranial venous drainage is different in craniosynostosis patients. ${ }^{6,9}$ Taking all of the above evidence together, we conclude that abnormality in cerebral venous dynamics is an important physiological feature of single-suture craniosynostosis. However, understanding the interaction between cerebral venous blood flow, cerebrospinal fluid (CSF) drainage, and ICP also requires consideration of anatomy. For example, the superficial venous drainage system, as reflected in the SSS, drains blood from the lateral aspects of the anterior portion of the cerebral hemispheres and collects CSF from the arachnoid granulations. The internal cerebral vein (ICV) is a component of the deep venous drainage system, and on each side of the brain it takes blood from the choroid plexus and thalamic and caudate nuclei. Therefore, in the current pilot investigation we have used Doppler ultrasound to examine cerebral venous flow velocity in the superficial and deep cerebral venous drainage systems to better characterize the complex intracranial venous physiology of craniosynostosis. Comparing both venous drainage systems enables us to examine the effect of craniosynostosis on the deep and superficial venous drainage system and, therefore, to evaluate the effect of corrective surgery on venous drainage and to identify possible targets to prevent ICHT.

\section{Methods}

This study was approved by our institution's medical ethics committee. Informed consent was obtained from all participants. Participants with syndromic and nonsyndromic craniosynostosis were recruited from craniosynostosis patients presenting to the Dutch craniofacial center in 2016. The healthy control group was also recruited at our center and comprised patients referred for nonsynostotic occipital plagiocephaly, metopic ridging, or nonsyndromic cleft lip.

\section{Patient Management}

Craniosynostosis patients were treated according to our
TABLE 1. Blood flow waveform categories as described by lkeda et al. ${ }^{11}$

\begin{tabular}{cc}
\hline Grade & Waveform \\
\hline 0 & Steady waveform; constant perfusion speed \\
\hline 1 & $\begin{array}{c}\text { Fluctuating waveform; minimum speed is never less than } \\
\text { half the maximum speed }\end{array}$ \\
\hline 2 & $\begin{array}{c}\text { Fluctuating waveform; minimum speed is less than half the } \\
\text { maximum speed, but never drops to } 0 \mathrm{~cm} / \mathrm{sec}\end{array}$ \\
\hline 3 & Fluctuating waveform; minimum speed drops to $0 \mathrm{~cm} / \mathrm{sec}$ \\
\hline
\end{tabular}

center's previously published treatment protocol..$^{14}$ Briefly, this meant that fronto-orbital advancement and remodeling was performed between 9 and 12 months of age for the following indications: metopic synostosis, unicoronal synostosis, Saethre-Chotzen's syndrome, and Muenke's syndrome. Sagittal synostosis patients were treated with springs, which were inserted at 5-6 months of age and removed approximately 12 weeks later. Patients with lambdoid synostosis, Apert's syndrome, or Crouzon's syndrome were treated with posterior decompression with the use of springs at around 5-6 months of age (with the springs removed 12 weeks later).

\section{Doppler Ultrasound Procedure and Analyses}

Prospective, transfontanelle ultrasound scans with Doppler studies were performed using an Esaote MyLab Twice ultrasound scanner. Scans were carried out at the first outpatient clinic visit and follow-up evaluation after each surgery. Controls underwent only 1 ultrasound study at the time of presentation to the outpatient clinic. During the ultrasound procedure, patients were positioned either supine or with the head of the bed elevated to a maximum of $30^{\circ}$. Studies were carried out when a child was quiet and at rest. Data from agitated or crying children were excluded because of the influence of heart rate variability and raised intrathoracic pressure on measurement of $\mathrm{SSS}_{\mathrm{v}}$ and mean venous blood flow velocity of the ICV $\left(\mathrm{ICV}_{\mathrm{v}}\right)$.

$\mathrm{ICV}_{\mathrm{v}}$ was measured in the sagittal plane using a convex ultrasound probe at $6.5 \mathrm{MHz}$ (or at $4.5 \mathrm{MHz}$ in those with larger skulls). As position and flow direction were the same in all patients and controls, we did not use any angle correction in the measurements. $\mathrm{SSS}_{\mathrm{v}}$ was measured in the coronal plane using a linear probe $(6.5 \mathrm{MHz}$ frequency) and an angle of $30^{\circ}$ to $45^{\circ}$. The Doppler range gate (2.2 $\mathrm{mm}$ ) was constant in all measurements.

All ultrasound and Doppler data were obtained by one of two observers (M.J.C. or P.D.) and digitally stored. (The interobserver agreement for mean $\mathrm{ICV}_{\mathrm{v}}$ and mean $\mathrm{SSS}_{\mathrm{v}}$, as assessed by intraclass correlation coefficient, was $>0.95$.) The ICV blood flow waveform produced by spectral analysis using image-processing software (Esaote MyLab) was scored using a previously described categorization (Table 1). ${ }^{11}$ Two observers (M.J.C. and R.d.G.) scored the waveform independently. Instances of disagreement between the scorers was resolved by open evaluation and agreed consensus. The evaluators' kappa statistics were 0.89 and 0.73 for the ICV and SSS waveforms, respectively. 
TABLE 2. Preoperative baseline characteristics and mean blood flow velocities

\begin{tabular}{lcrrr}
\hline \multirow{2}{*}{ Variable } & \multicolumn{4}{c}{ Mean \pm SEM (no. of measurements) } \\
\cline { 2 - 5 } & Nonsyndromic Craniosynostosis & Syndromic Craniosynostosis & All Craniosynostosis Patients & Controls \\
\hline Age in mos & $4.04 \pm 0.57(28)$ & $2.71 \pm 0.72(6)$ & $3.81 \pm 0.49(34)$ & $6.04 \pm 0.42(28)$ \\
\hline OFC $^{*}$ & $+0.62 \pm 0.24(28)$ & $-0.32 \pm 0.79(6)$ & $+0.45 \pm 0.24(34)$ & $+0.15 \pm 0.25(22)$ \\
\hline $\mathrm{SSS}_{\mathrm{v}}$ in cm/sec & $7.80 \pm 0.51(23)$ & $6.66 \pm 0.71(6)$ & $7.57 \pm 0.44(29)$ & $11.31 \pm 1.06(26)$ \\
\hline $\mathrm{ICV}_{\mathrm{v}}$ in cm/sec & $10.00 \pm 0.34(22)$ & $8.57 \pm 0.76(5)$ & $9.74 \pm 0.33(27)$ & $9.68 \pm 0.29(26)$ \\
\hline
\end{tabular}

* OFC in standard deviations compared to the national normal values.

\section{Statistical Analyses}

The sample size for our pilot study was based on previous guidelines ${ }^{16}$ and our center's medical ethics committee's recommendations. The statistical analyses assumed normal distribution for $\mathrm{ICV}_{\mathrm{v}}$ and $\mathrm{SSS}_{\mathrm{v}}$ data. A multivariate analysis of variance (MANOVA) test was performed to assess the effect of craniosynostosis on $\mathrm{SSS}_{\mathrm{v}}$ and $\mathrm{ICV}_{\mathrm{v}}$. The chi-square test was used for assessment of waveform categorical data. Finally, in the comparisons of pre- to postoperative change, we used the preoperative data along with the data from after the last (or most recent) operation. Post hoc nonparametric testing (Kruskal-Wallis or Wilcoxon signed-rank test) was performed when appropriate.

\section{Results}

We recruited 34 craniosynostosis patients, including 14 patients with sagittal synostosis, 11 with metopic synostosis, 2 with unicoronal synostosis, 1 with lambdoid synostosis, 1 with Saethre-Chotzen's syndrome, 3 with Muenke's syndrome, and 2 with Crouzon's syndrome. Postoperatively, we were able to obtain ultrasound scans in $22(65 \%)$ of these 34 patients ( 8 with sagittal suture synostosis, 9 with metopic synostosis, 1 with lambdoid synostosis, 1 with Saethre-Chotzen's syndrome, 2 with Muenke's syndrome, and 1 with Crouzon's syndrome). The control group comprised 28 patients ( 24 with nonsynostotic occipital plagiocephaly or metopic ridging, 2 with cleft lip, and 2 unaffected twin siblings of craniosynostosis patients).

None of the patients with craniosynostosis had papilledema at the time of initial assessment. One patient with Muenke's syndrome developed papilledema after the preoperative ultrasound study, and for this reason she underwent posterior cranial vault decompression. Additionally, 1 patient with Crouzon's syndrome developed papilledema after the first ultrasound. At the time of the postoperative ultrasound study the papilledema was resolving in both cases but had not completely disappeared. None of the other patients had papilledema at the postoperative assessment.

\section{Preoperative ICV $\mathrm{IV}_{\mathrm{v}}$ and $\mathrm{SSS}_{\mathrm{v}}$}

Table 2 summarizes the initial findings in the 3 study groups (patients with nonsyndromic or syndromic craniosynostosis and controls). The age distribution differed significantly between groups (Kruskal-Wallis test, $\mathrm{p}<0.001$ ). Post hoc testing showed no significant difference with regard to age at ultrasound between the syndromic and nonsyndromic craniosynostosis groups (Mann-Whitney
U-test, $p=0.24$ ), but it did show a significant difference between the nonsyndromic group and controls (MannWhitney U-test, $\mathrm{p}=0.001$ ). There was no significant between-groups difference in occipitofrontal head circumference (OFC) (ANOVA, $\mathrm{p}=0.20)$.

We performed a MANOVA analysis to test whether there were significant differences with regard to venous flow velocity between craniosynostosis patients and controls, correcting for age at ultrasound and OFC. This analysis showed significantly lower venous blood flow velocity in the $\mathrm{SSS}_{\mathrm{v}}$ in craniosynostosis patients compared with controls (Table 3). Age at ultrasound and OFC were not significant contributors to this effect. Additional testing did not show statistically significant differences between nonsyndromic and syndromic craniosynostosis patients after correction for age at ultrasound and OFC.

\section{Venous Blood Flow Waveform}

Preoperative cerebral venous blood flow waveform scores are shown in Table 4 . The chi-square test did not show any significant differences in distribution among the different groups for the 2 measurements.

\section{Postoperative Blood Flow Velocity}

Preoperative and postoperative cerebral venous blood flow velocities of the SSS were gained in 15 patients: 4 patients with scaphocephaly, 6 with trigonocephaly, 1 with lambdoid synostosis, 1 with Crouzon's syndrome, 1 with Saethre-Chotzen's syndrome, and 2 with Muenke's syndrome. A related-samples Wilcoxon signed-rank test showed a significant increase in $\mathrm{SSS}_{\mathrm{v}}$ postoperatively (median $7.25 \mathrm{~cm} / \mathrm{sec}$ [IQR $6.75-8.95 \mathrm{~cm} / \mathrm{sec}$ ] vs $10.20 \mathrm{~cm} / \mathrm{sec}$ [IQR 8.95-12.20 cm/sec], $\mathrm{p}=0.023)$ The $\mathrm{ICV}_{\mathrm{v}}$ remained unchanged (median $9.90 \mathrm{~cm} / \mathrm{sec}$ [IQR 7.95-10.98 cm/sec] vs $10.15 \mathrm{~cm} / \mathrm{sec}$ [IQR $8.00-11.35 \mathrm{~cm} / \mathrm{sec}$ ], p=0.68). Fig.

TABLE 3. MANOVA correcting for age at time of ultrasound and OFC

\begin{tabular}{|c|c|c|c|c|c|}
\hline \multirow[b]{2}{*}{ Variable } & \multicolumn{2}{|c|}{ Mean \pm SEM } & \multirow[b]{2}{*}{$\mathrm{F}$} & \multirow[b]{2}{*}{ df } & \multirow{2}{*}{$\begin{array}{c}p \\
\text { Value }\end{array}$} \\
\hline & Craniosynostosis & Controls & & & \\
\hline SSS $_{v}$ & $7.37 \pm 0.33$ & $11.51 \pm 1.13$ & 7.253 & 1 & $0.009^{*}$ \\
\hline $\mathrm{ICV}_{\mathrm{v}}$ & $9.74 \pm 0.34$ & $9.30 \pm 0.30$ & 0.180 & 1 & 0.612 \\
\hline
\end{tabular}

$\mathrm{df}=$ degree of freedom.

Velocities are presented in centimeters/second. Design: intercept + age at ultrasound + OFC + craniosynostosis. Adjusted $\mathrm{R}^{2}=0.25$.

* Statistically significant. 
TABLE 4. Distribution of preoperative blood flow waveform grades in patients with nonsyndromic or syndromic craniosynostosis and controls

\begin{tabular}{cccc}
\hline Variable & $\begin{array}{c}\text { Nonsyndromic } \\
\text { Craniosynostosis }\end{array}$ & $\begin{array}{c}\text { Syndromic } \\
\text { Craniosynostosis }\end{array}$ & Controls \\
\hline ICV & & & \\
\hline Grade 0 & 5 & 0 & 3 \\
\hline Grade 1 & 17 & 5 & 23 \\
\hline Grade 2 & 0 & 0 & 0 \\
\hline Grade 3 & 0 & 0 & 0 \\
\hline Total & 22 & 5 & 26 \\
\hline SSS & & & \\
\hline Grade 0 & 7 & 1 & 5 \\
\hline Grade 1 & 12 & 4 & 19 \\
\hline Grade 2 & 4 & 1 & 2 \\
\hline Grade 3 & 0 & 0 & 26 \\
\hline Total & 23 & 6 & \\
\hline
\end{tabular}

Values are numbers of patients. The preoperative venous blood flow waveform grades are based on the scoring system described in Table 1. No significant differences were found for ICV ( $p=0.77)$ or SSS waveform $(p=0.62)$ using the chi-square test.

1 shows patient-specific pre- to postoperative change of the $\mathrm{SSS}_{\mathrm{v}}$.

\section{Discussion}

This study assessed cerebral venous blood flow velocity and blood flow waveform in patients with craniosynostosis compared with controls using Doppler ultrasound. Before surgery, patients with craniosynostosis showed lower $\mathrm{SSS}_{\mathrm{v}}$ compared with controls, that increased postoperatively. There was no difference in $\mathrm{ICV}_{\mathrm{v}}$ at any time, and blood flow waveform characteristics were similar in both cases and controls.

Previous studies have reported on the interaction between hydrocephalus and hydrodynamic and hemodynamic pressures (i.e., raised ICP and $\mathrm{P}_{\mathrm{SSS}}$ ) in various patient groups. ${ }^{10,15,16,19}$ For example, in children with achondroplasia and hydrocephalus, cine phase-contrast MRI shows reduced $\mathrm{SSS}_{\mathrm{v} \cdot}{ }^{10}$ Hirabuki et al. hypothesized that the reduced cerebral venous blood flow, as indicated by reduced $\mathrm{SSS}_{\mathrm{v}}$, was the result of venous outflow obstruction..$^{10}$ In our current findings, we have taken measurements from both the superficial and deep cerebral venous drainage systems as a test of the anatomy before and after any potential point of venous constriction or compression. The resulting data indicate that premature closure of cranial sutures may, in itself, be related to decreased $\mathrm{SSS}_{\mathrm{v}}$ and thus reduced cerebral venous drainage from the superficial system, i.e., at a point proximate to the confluence of the straight sinus and SSS. In contrast to these observations, Mursch et al. found higher $\mathrm{SSS}_{\mathrm{v}}$ in craniosynostosis patients, ${ }^{15}$ it should, however, be noted that their measurements were made at the site of venous constriction/compression. Of interest, de Souza and Pinto showed that the diameter of the SSS is related to sagittal suture growth. ${ }^{4}$ These findings, together with our own, strengthen the hypothesis that cerebral venous out- flow obstruction due to venous constriction or compression is caused by the presence of a synostotic suture. Consistent with this idea is our observation that decreased $\mathrm{SSS}_{\mathrm{v}}$ is also found in single-suture craniosynostosis patients (Table 2); until now, cerebral venous hypertension has been considered an attribute of syndromic craniosynostosis. ${ }^{6,9}$ In fact, we think that this physiology may be important in the etiology of ICHT in unisutural craniosynostosis patients, particularly as OSA, Chiari malformation type I, and hydrocephalus are not found in this patient group. Furthermore, the postoperative increase in $\mathrm{SSS}_{\mathrm{v}}$ may also reflect that venous obstruction/compression caused by the synostotic suture has been relieved and resistance into venous outflow in the superficial drainage system has been lowered. Since we only performed postoperative analyses in 15 patients, these findings should be confirmed in a larger study.

In regard to the characteristics of the cerebral venous waveform in craniosynostosis, Mursch et al. previously reported that such patients had different SSS pulsatility measurements (i.e., pulsatility index and resistance index). ${ }^{15}$ We could not reproduce these findings when using a system of scoring venous blood flow waveform profiles. Taken together with the above discussion of $\mathrm{SSS}_{\mathrm{v}}$, this observation suggests that we may have been seeing patients early in the course of uncorrected natural history; that is, at an early stage of pathology when there is premature suture fusion with an effect on $\mathrm{SSS}_{\mathrm{v}}$ but pulsatility remains unchanged. The state in which craniosynostosis influences both $\mathrm{SSS}_{\mathrm{v}}$ and SSS waveform pulsatility most likely represents a more severe or later stage.

There are some limitations in this study that need to be considered. First, we have little comparative data. Even though the cranial venous outflow of patients with craniosynostosis has been a subject of research over the past decade, we have only one quantitative study of $\mathrm{SSS}_{\mathrm{y}}$, until now. The present study was designed as a pilot project to explore potential effects of craniosynostosis on the superficial and deep cerebral venous drainage systems, and we hope that our findings will stimulate research in other clinical centers. Second, in accordance with our institution's medical research ethics advice for pilot studies, we could only recruit up to 15 patients in each diagnostic group, which, at this preliminary stage, limits the generalization of our findings. Third, postoperative analyses were limited by the presence of closure of the anterior fontanelle-the radiological "window" for examining the SSS and ICV. We have no control over this limitation, but in the future dynamic cerebral MR venography may provide useful information. Fourth, flow velocity is not equal to flow volume. In this study we showed that there is a lower flow velocity in the SSS, but it is not yet proven that this also means a lower flow volume. However, we do believe a lower flow velocity is more likely to represent a lower flow volume in this case, especially given the flow velocity increase postoperatively. Last, technical components of Doppler ultrasound studies have the potential to add to variability, e.g., angle of insonation, patient activity, and positioning. We have limited these potential technical effects by standardizing our approach and excluding data that are inadequate (e.g., because patients were restless or moving). 


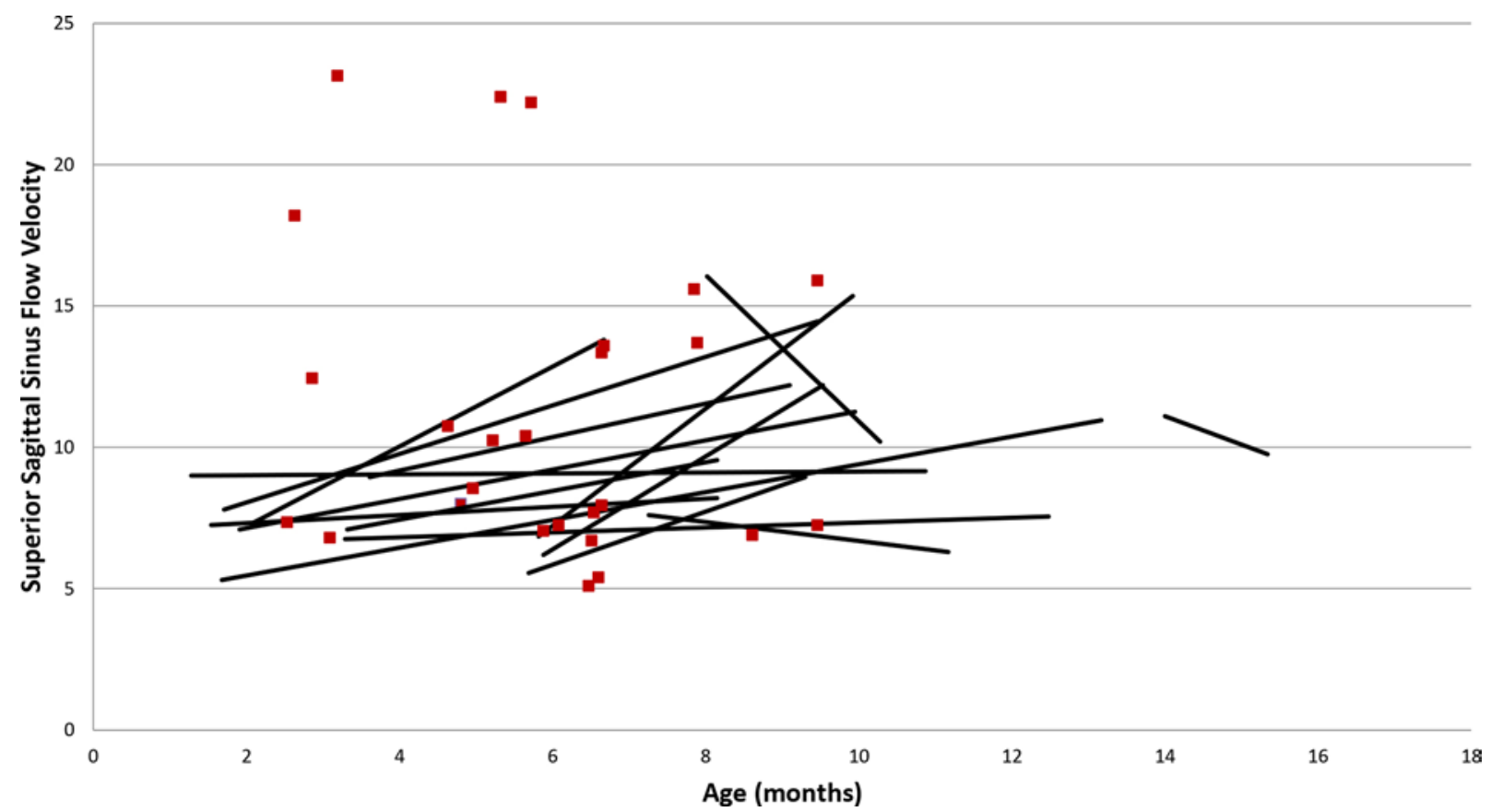

FIG. 1. Preoperative to postoperative change in superior sagittal sinus flow velocity of the patients. Each black line indicates the data from 1 patient. The red squares represent point data from controls. See text for details. Figure is available in color online only.

Therefore, considering the results of the present study and the above limitations, our hypothesis is that cerebral venous drainage and outflow has a role in the etiology of ICHT, even in single-suture craniosynostosis patients. This finding should be confirmed in future studies, which should also explore potential differences between the different types of craniosynostosis. It would also be interesting to test whether patients with an affected suture in the midline (i.e., metopic or sagittal synostosis) show a more profound effect of suture closure on $\mathrm{SSS}_{\mathrm{v}}$ compared with the other subtypes of craniosynostosis. In addition, the effect of surgery should be evaluated, especially the possible hierarchy in severity (i.e., closed suture with decreased $\mathrm{SSS}_{\mathrm{v}}$ vs decreased $\mathrm{SSS}_{\mathrm{v}}$ with abnormal venous waveform).

\section{Conclusions}

This pilot study of cerebral venous outflow patterns in craniosynostosis patients shows that premature closure of cranial sutures is associated with decreased $\mathrm{SSS}_{\mathrm{v}}$, but not $\mathrm{ICV}_{\mathrm{v}}$ - that is, an effect on the superficial venous drainage rather than deep venous drainage. Further Doppler ultrasound studies are needed, not only to confirm the current findings, but also to test the hypothesis that at an early stage of craniosynostosis pathology $\mathrm{SSS}_{\mathrm{v}}$ is abnormal while pulsatility is normal, and that abnormalities in both $\mathrm{SSS}_{\mathrm{v}}$ and the superficial venous waveform reflect a more advanced stage of evolution in suture closure.

\section{Acknowledgments}

This study was funded by Fonds NutsOhra (grant no. FNO 1404-052).

\section{References}

1. Cornelissen M, Ottelander Bd, Rizopoulos D, van der Hulst $\mathrm{R}$, Mink van der Molen A, van der Horst C, et al: Increase of prevalence of craniosynostosis. J Craniomaxillofac Surg 44:1273-1279, 2016

2. Cornelissen MJ, Loudon SE, van Doorn FE, Muller RP, van Veelen MC, Mathijssen IM: Very low prevalence of intracranial hypertension in trigonocephaly. Plast Reconstr Surg 139:97e-104e, 2017

3. de Jong T, Bannink N, Bredero-Boelhouwer HH, van Veelen ML, Bartels MC, Hoeve LJ, et al: Long-term functional outcome in 167 patients with syndromic craniosynostosis; defining a syndrome-specific risk profile. J Plast Reconstr Aesthet Surg 63:1635-1641, 2010

4. de Souza RB, Pinto FC: Could craniometric measurements explain the growth of the superior sagittal sinus? Pediatr Neurosurg 48:225-228, 2012

5. Di Rocco C, Frassanito P, Massimi L, Peraio S: Hydrocephalus and Chiari type I malformation. Childs Nerv Syst 27:1653-1664, 2011

6. Florisson JM, Barmpalios G, Lequin M, van Veelen ML, Bannink N, Hayward RD, et al: Venous hypertension in syndromic and complex craniosynostosis: the abnormal anatomy of the jugular foramen and collaterals. J Craniomaxillofac Surg 43:312-318, 2015

7. Florisson JM, van Veelen ML, Bannink N, van Adrichem LN, van der Meulen JJ, Bartels MC, et al: Papilledema in isolated single-suture craniosynostosis: prevalence and predictive factors. J Craniofac Surg 21:20-24, 2010

8. Gault DT, Renier D, Marchac D, Jones BM: Intracranial pressure and intracranial volume in children with craniosynostosis. Plast Reconstr Surg 90:377-381, 1992

9. Hayward R: Venous hypertension and craniosynostosis. Childs Nerv Syst 21:880-888, 2005

10. Hirabuki N, Watanabe Y, Mano T, Fujita N, Tanaka H, Ueguchi T, et al: Quantitation of flow in the superior sagittal sinus performed with cine phase-contrast MR imaging of healthy 
and achondroplastic children. AJNR Am J Neuroradiol 21:1497-1501, 2000

11. Ikeda T, Amizuka T, Ito Y, Mikami R, Matsuo K, Kawamura $\mathrm{N}$, et al: Changes in the perfusion waveform of the internal cerebral vein and intraventricular hemorrhage in the acute management of extremely low-birth-weight infants. Eur J Pediatr 174:331-338, 2015

12. Maltese G, Tarnow P, Wikberg E, Bernhardt P, Lagerlöf JH, Tovetjärn R, et al: Intracranial volume before and after surgical treatment for isolated metopic synostosis. J Craniofac Surg 25:262-266, 2014

13. Marucci DD, Dunaway DJ, Jones BM, Hayward RD: Raised intracranial pressure in Apert syndrome. Plast Reconstr Surg 122:1162-1170, 2008

14. Mathijssen IM: Guideline for care of patients with the diagnoses of craniosynostosis: working group on craniosynostosis. J Craniofac Surg 26:1735-1807, 2015

15. Mursch K, Enk T, Christen HJ, Markakis E, Behnke-Mursch $\mathrm{J}$ : Venous intracranial haemodynamics in children undergoing operative treatment for the repair of craniosynostosis. A prospective study using transcranial colour-coded duplex sonography. Childs Nerv Syst 15:110-118, 1999

16. Olivero WC, Rekate HL, Chizeck HJ, Ko W, McCormick JM: Relationship between intracranial and sagittal sinus pressure in normal and hydrocephalic dogs. Pediatr Neurosci 14:196-201, 1988

17. Renier D, Lajeunie E, Arnaud E, Marchac D: Management of craniosynostoses. Childs Nerv Syst 16:645-658, 2000

18. Renier D, Sainte-Rose C, Marchac D, Hirsch JF: Intracranial pressure in craniostenosis. J Neurosurg 57:370-377, 1982

19. Sainte-Rose C, LaCombe J, Pierre-Kahn A, Renier D, Hirsch JF: Intracranial venous sinus hypertension: cause or consequence of hydrocephalus in infants? J Neurosurg 60:727736,1984

20. van Veelen MC, Jippes M, Carolina JA, de Rooi J, Dirven $\mathrm{CM}$, van Adrichem LN, et al: Volume measurements on three-dimensional photogrammetry after extended strip versus total cranial remodeling for sagittal synostosis: a comparative cohort study. J Craniomaxillofac Surg 44:1713-1718, 2016

\section{Disclosures}

The authors report no conflict of interest concerning the materials or methods used in this study or the findings specified in this paper.

\section{Author Contributions}

Conception and design: Cornelissen, Doerga, Lequin, Govaert, Mathijssen, Dudink, Tasker. Acquisition of data: Cornelissen, de Goederen, Doerga, Cuperus. Analysis and interpretation of data: Cornelissen, de Goederen, Dudink, Tasker. Drafting the article: Cornelissen, de Goederen, Tasker. Critically revising the article: Doerga, Cuperus, van Veelen, Lequin, Govaert, Mathijssen, Dudink, Tasker. Reviewed submitted version of manuscript: Doerga. Statistical analysis: Cornelissen, de Goederen, Tasker. Administrative/technical/material support: Cuperus, van Veelen, Lequin. Study supervision: van Veelen, Lequin, Mathijssen, Tasker.

\section{Supplemental Information}

\section{Previous Presentations}

Portions of this paper were previously published in Dr. Cornelissen's thesis, "Unisutural craniosynostosis: simple or complex?" submitted as partial fulfillment of the PhD degree conferred on October 13, 2017, by Erasmus University, Rotterdam, The Netherlands.

\section{Correspondence}

Martijn J. Cornelissen: Sophia Children's Hospital, Erasmus MC, Rotterdam, The Netherlands.m.cornelissen@erasmusmc.nl. 